\section{Patient Safety and Satisfaction with Electroacupuncture in a Teaching Clinic: A Survey of Interns and Patients}

\author{
Jennifer Brett ${ }^{1 *}$ and Medeya Tsnobiladze ${ }^{2}$ \\ ${ }^{1}$ Acupuncture Institute, University of Bridgeport, Bridgeport CT, USA \\ ${ }^{2}$ Physician Assistant Institute, University of Bridgeport, Bridgeport CT, USA
}

\begin{abstract}
Acupuncture, including electroacupuncture, is an important modality in Traditional Chinese Medicine (TCM). Acupuncture has been increasingly used in Europe and North America in the past forty years. Its widening acceptance demands continual safety assessment. This pilot study evaluated the frequency and severity of Adverse Events (AE) for electroacupuncture in a teaching clinic.

Needle-only acupuncture has a documented high patient satisfaction rate and low Adverse Event (AE) rate.

Our study demonstrated that the satisfaction with and safety of electroacupuncture and needle-only acupuncture are similar.

Keywords: Acupuncture; Electro-acupuncture; Patient satisfaction; Safety
\end{abstract}

\section{Description}

The safety of needle acupuncture is well established. Early reviews of the literature include those by Ernst and White, and Lao who conclude: "The risk of serious events occurring in association with acupuncture is very low, below that of many common medical treatments" [1]. "Acupuncture performed by trained practitioners using Clean Needle Techniques [XE "Clean Needle Technique (CNT)"] is a generally safe procedure" [2].

Lao et al., reviewed literature covering the years 1965-1999. "Over the 35 years, 202 incidents were identified in 98 relevant papers reported from 22 countries. Types of complications included infections [XE "infection"], and organ, tissue, and nerve injury [XE "injury: nerve injury"]. Adverse effects included cutaneous [XE "cutaneous"] disorders, hypotension, fainting [XE "fainting"], and vomiting" [2].

White reviewed a significant body of published evidence regarding $\mathrm{AE}$ associated with acupuncture offering a numerical

\footnotetext{
*Corresponding author: Jennifer Brett, Acupuncture Institute, University of Bridgeport, Bridgeport CT, USA, Tel: +1 2035764122; E-mail: jbrett@bridgeport.edu
}

Citation: Brett J, Tsnobiladze M (2015) Patient Safety and Satisfaction with Electroacupuncture in a Teaching Clinic: A Survey of Interns and Patients. J Altern Complement Integr Med 1: 001.

Received: June 06, 2015; Accepted: July 03, 2015; Published: July 20, 2015 value of $\mathrm{AE}$ associated with acupuncture treatments. "According to the evidence from 12 prospective studies which surveyed more than a million treatments, the risk of a serious adverse event [XE "Adverse Events: Serious (SAE)"] with acupuncture is estimated to be 0.05 per 10,000 treatments, and 0.55 per 10,000 individual patients. The risk of serious events occurring in association with acupuncture is very low, below that of many common medical treatments [3].

Later prospective studies conclude similarly that the vast majority of adverse effects are minor and require little or no treatment. Melchart et al., [4] studied 97,733 patients who were followed over time and found 7\% reported adverse effects including needle site pain (3\%), bruising (3\%) and bleeding (1\%) with rare serious adverse effects including pneumothorax. Park et al., [5] studied 2226 patients over 5 weeks of acupuncture treatments and found only 99 adverse events during that time (4.5\%). The most common were bleeding [XE "bleeding"], /bruising [XE "bruising"] $(2.7 \%)$ and needle site pain $(2.7 \%)$. The most likely moderately severe side effect was nerve injury [XE "injury: nerve injury"] $(0.31 \%)$ described as temporary paresthesia [XE "paresthesia"] which disappeared within 1 week. No serious adverse events were experienced by any patients during this study. Witt et al., [6] observed 229,230 patients receiving, on average, ten treatments for common complaints such as pain and allergies. Of these, 19,726 reported adverse effects (8.6\%). Common events again included bleeding/ bruising $(6.14 \%)$, fatigue $(1.15 \%)$, headache $(0.52 \%)$, pain including pain at the site of needle insertion (1.7\%), and aggravation of symptoms [XE "aggravation of symptoms"] (0.31\%). Serious adverse events [XE "Adverse Events: Serious (SAE)"] included 2 cases of pneumothorax [XE "pneumothorax"] and 31 cases of nerve injury $(0.014 \%), 31$ instances of local infections [XE "infection"] at the acupuncture insertion points were reported $(0.014 \%)$ and 5 systemic infections were reported.

In the most recent comprehensive review of adverse events associated with acupuncture, moxibustion and cupping [XE "cupping"], Xu et al., [7] found that between 2000 and 2011 (12 years) "117 reports of 308 AEs from 25 countries and regions were associated with acupuncture (294 cases), moxibustion (4 cases), or cupping (10 cases)". Serious organ and tissue injury continue to be reported but are rare. These authors note a significant reduction in the number of infections compared to earlier reports.

While needle only acupuncture has been shown to have a high patient satisfaction rate $[8,9]$ and low adverse event rate $[1-7,10,11]$, there is a paucity of information about electroacupuncture. We designed this prospective study in order to evaluate whether the satisfaction with and safety of the two types of acupuncture are similar.

There are very few studies of the potential adverse effects of Electro-Acupuncture (EA). One recent review of the literature from 1979-2010 [7] found only 44 incidences of AE reported during that time frame. Another review of the Chinese literature [11] similarly found few adverse events associated with electroacupuncture. While a number of the $\mathrm{AE}$ were probably associated with the acupuncture needling (faintness, hyperventilation) a few were associated with the 
application of an electrical current (electrical injury, atrioventricular block, dislocation of the wrist joint from muscle spasm) [7-11].

In order to match earlier studies on acupuncture safety, such as the SAFA study by White et al., [10] we defined adverse events to be any ill effect that is unintended and non-therapeutic. This included errors, non-serious events such as minor bleeding, and any serious events.

Our aim was to determine if the rate of errors, incidences and adverse events are similar for electroacupuncture when compared to needle only acupuncture in a teaching clinic setting.

\section{Methods}

Patients in the University of Bridgeport Acupuncture Institute teaching clinic during a six month period were recruited and randomly assigned to participate in the study and survey. On each shift with an IRB-approved clinical supervisor three return patients chosen randomly by time slot for their appointments were offered the electroacupuncture treatment and follow up survey; two other return clinic patients were assigned to participate in the survey following needle only acupuncture treatments and satisfaction survey. New patients were not recruited for the study. Those patients who would be receiving care other than needle acupuncture (e.g., gua sha, cupping, moxibustion) were excluded from the study. Patients who had pacemakers or other implanted electrical devices were excluded from the study. All selected patients in both arms of the study were advised of the study through a consent form and given the opportunity to refuse to participate.

All patients received the usual care (intake, tongue and pulse analysis, assessment, TCM treatment). Acupuncture needling was performed such that "de qi" response was elicited for all participating patients. ("De qi" is a sensation of the needle being pulled or grabbed felt by the practitioner; and is often described as a tingling or aches by the patient). For clinic patients randomly assigned the electroacupuncture arm of the study and for whom there were no contra-indications for the treatment, two pairs of peripheral acupuncture points on the extremities were stimulated with electricity. The chosen points were needled, a de qi response was achieved with standard needle manipulation and then electrical stimulation was applied at a pulse rate of 50 or higher and at an amplitude such that the patient was aware of the stimulation but without significant pain or any muscle spasms. Needles were retained for 20-30 minutes on all patients receiving electroacupuncture or needle-only acupuncture. At the conclusion of the treatment, the patients completed an online survey about their experience and any side effects before leaving the clinic.

Student interns filled out an online survey about their experience and any errors or adverse events. Adverse events and errors that were specifically identified on the survey form included: retained needle (a practitioner error in which a needle is inadvertently left in a patient at the end of the visit), lost or dropped needle, bleeding more than 10 seconds at any acupuncture site, swelling at any site, cramping, needle site pain (not including de qi), nausea, sweating, feeling faint, fainting, and drowsiness.

111 patients received acupuncture with electrical stimulation; 52 received acupuncture without electrical stimulation. All patients in the study completed online "snap" surveys of their care and satisfaction. Additionally, student interns completed 135 surveys about their experience with patient treatment and any adverse events that occurred during treatment.
In this study, supervising clinicians were asked to complete a separate report form in the event of any serious adverse events. No such report forms were received.

A total of 163 patient surveys were collected over a 6 month period from May 2014 to December 2014. 135 student intern surveys were completed during that same time period. No serious adverse event report forms were received during the six month period.

Approval for the study was obtained from the University of Bridgeport Institutional Review Board. All patients in the study completed a written consent form for study participation prior to receiving acupuncture or electroacupuncture.

\section{Results}

Patients recruited for both arms of the study had similar characteristics. There was a preponderance of females in the study with the average age of those recruited being over 50 years (Table 1).

\begin{tabular}{|c|c|c|c|}
\hline & $\begin{array}{c}\text { Patient Report } \\
\text { With E-Stim }\end{array}$ & $\begin{array}{c}\text { Patient Report } \\
\text { No E-Stim }\end{array}$ & $\begin{array}{c}\text { Total in } \\
\text { Study }\end{array}$ \\
\hline Male & $44(39.6 \%)$ & $10(19.2 \%)$ & $54(33.1 \%)$ \\
\hline Female & $67(60.4 \%)$ & $42(80.8 \%)$ & $109(66.8 \%)$ \\
\hline Average Age & 54 & 52 & \\
\hline Race/Ethnicity & $5(4.5 \%)$ & $3(5.8 \%)$ & $8(4.9 \%)$ \\
\hline Asian & $1(0.9 \%)$ & $0(0.0 \%)$ & $1(0.6 \%)$ \\
\hline Pacific Islander & $5(4.5 \%)$ & $2(3.8 \%)$ & $7(4.3 \%)$ \\
\hline Black/African American & $1(0.9 \%)$ & $0(0.0 \%)$ & $1(0.6 \%)$ \\
\hline $\begin{array}{c}\text { American Indian/Alaska } \\
\text { Native }\end{array}$ & $78(70.9 \%)$ & $33(63.5 \%)$ & $111(68.5 \%)$ \\
\hline $\begin{array}{c}\text { White (Not Hispanic or } \\
\text { Latino) }\end{array}$ & $17(15.5 \%)$ & $12(23.1 \%)$ & $29(17.9 \%)$ \\
\hline $\begin{array}{c}\text { Hispanic or Latino (All } \\
\text { Races) }\end{array}$ & $3(2.7 \%)$ & $2(3.8 \%)$ & $5(3.1 \%)$ \\
\hline Other; did not want to report & & & \\
\hline
\end{tabular}

Table 1: Demographics of the patients in the electroacupuncture and needle only arms of the study.

Note: All demographics were self-reported by the participants in the confidential online survey instrument utilized in this study.

In order to more thoroughly compare the needle-only acupuncture adverse events with the electroacupuncture side effects, we compared our results with the McPherson survey [9] and the SAFA study [10]. In those two studies both positive non-specific effects (feeling energized and feeling relaxed) as well as adverse events were noted. Since we based our study on these two studies, we compared our outcomes with those two previous studies in table 2 (Figure 1).

\section{Clinical Significance}

Patients receiving electroacupuncture were statistically less likely to feel relaxed than those with manual acupuncture alone $(\mathrm{P}=0.001)$ (Table 3). This may be due to patients completing a consent form that listed any number of possible side effects from electrical stimulation or could be attributed to feeling apprehension towards "electricity".

The difference in the level of reported cramping as also statistically significant, $\mathrm{p}=0.017$. 
Citation: Brett J, Tsnobiladze M (2015) Patient Safety and Satisfaction with Electroacupuncture in a Teaching Clinic: A Survey of Interns and Patients. J Altern Complement Integr Med 1: 001 .

- Page 3 of 5 •

\begin{tabular}{|l|c|c|c|c|c|c|}
\hline \multicolumn{1}{|c|}{ Event } & $\begin{array}{c}\text { Patient Report With } \\
\text { E-Stim }\end{array}$ & $\begin{array}{c}\text { Patient Report No } \\
\text { E-Stim }\end{array}$ & Effect Size (Cohen) & Intern Report & $\begin{array}{c}\text { White SAFA Study } \\
(\mathbf{2 0 0 1})\end{array}$ & $\begin{array}{c}\text { McPherson Study } \\
\text { (2005) }\end{array}$ \\
\hline Cramping & $11.20 \%$ & $4.40 \%$ & 0.257 & $3.60 \%$ & $0.50 \%$ \\
\hline Needle site pain & $20.20 \%$ & $15.60 \%$ & 0.087 & $4.80 \%$ & $1.10 \%$ & $12 \%$ \\
\hline Nausea & $0.00 \%$ & $0.00 \%$ & NA & $0.00 \%$ & $1.20 \%$ \\
\hline Feeling faint & $1.10 \%$ & $0.00 \%$ & NA & Not reported & $0.29 \%$ & $2.60 \%$ \\
\hline Sweating & $3.40 \%$ & $2.20 \%$ & 0.072 & $1.20 \%$ & $0.10 \%$ & $0.80 \%$ \\
\hline Drowsiness & $11.20 \%$ & $13.30 \%$ & 0.093 & $1.20 \%$ & $0.30 \%$ & $0.60 \%$ \\
\hline Feeling relaxed & $78.70 \%$ & $86.70 \%$ & 0.274 & $62.70 \%$ & & $79.10 \%$ \\
\hline Feeling energized & $48.30 \%$ & $44.40 \%$ & 0 & $19.30 \%$ & & $32.70 \%$ \\
\hline $\begin{array}{l}\text { Nervous or apprehensive about the } \\
\text { treatment }\end{array}$ & $13.50 \%$ & $17.90 \%$ & & & & \\
\hline $\begin{array}{l}\text { Patient identified likelihood of returning for } \\
\text { additional treatments? }\end{array}$ & $91 \%$ & $88 \%$ & & & & \\
\hline Total Negative Reactions: & $38 \%$ & $30 \%$ & & & & \\
\hline Bleeding or hematoma & & & & & \\
\hline
\end{tabular}

Table 2: Adverse Event/Incidence Reports.

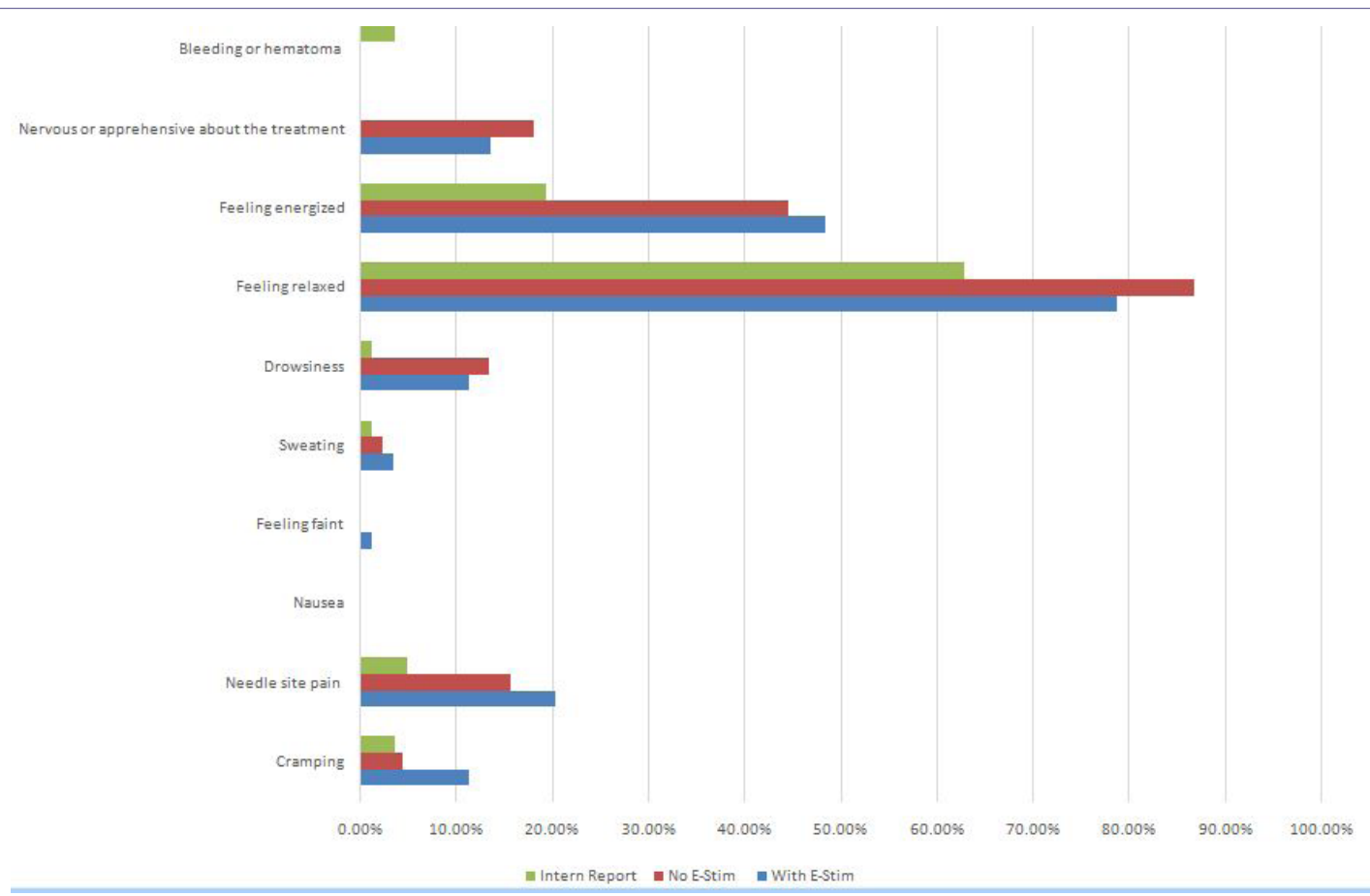

Figure 1: Comparison of AE reported by patients receiving E-Stim, patients receiving no E-stim, \& intern reports of AE.

\section{Discussion}

In this study both the patients receiving electrical stimulation acupuncture and non-electrical stimulation acupuncture were equally satisfied with their treatments. There were no significant adverse events associated with either arm of the study (Table 4 and Figure 2).

In the electrical stimulation acupuncture group there was a trend toward more pain and cramping reported than in the non-electrical stimulation acupuncture group. Of these two findings, only the cramping showed an effect size of greater than 0.2 and therefore only a small clinical difference $(P=0.017)$. It is possible that this is a true finding, given that electrical stimulation is known to cause muscle cramping at certain settings. It is also possible that this was due to the choice of patients that received electrical stimulation acupuncture (supervising clinicians had the option to identify whether the patients needed electrical stimulation or not), or the heavier gauge needles and deeper needle placement that are often used to support the electrodes utilized for the electrical stimulation.

Those patients not receiving electrical stimulation did trend toward being more relaxed about the procedure than those who participated in the electrical stimulation arm of the study $(\mathrm{P}=0.001)$. This difference was small and had no impact on patient satisfaction.

There were no other reported adverse reactions that were statistically different between the two groups.

Of note, there was a significant discrepancy between the patients' reports of needle site pain, cramping and drowsiness compared to the interns' impressions of the outcomes. Whether this is due to the nature 
Citation: Brett J, Tsnobiladze M (2015) Patient Safety and Satisfaction with Electroacupuncture in a Teaching Clinic: A Survey of Interns and Patients. J Altern Complement Integr Med 1: 001.

- Page 4 of 5 •

\begin{tabular}{|c|c|}
\hline Errors & Events \\
\hline $3(3.6 \%)$ & Visible bruising \\
\hline $1(1.2 \%)$ & Bleeding lasting 10 seconds or longer \\
\hline $2(2.4 \%)$ & Dropped needle \\
\hline $3(3.6 \%)$ & Lost needle (needle in count did not correlate with needles removed) \\
\hline $0(0.0 \%)$ & Trouble removing a needle \\
\hline $1(1.3 \%)$ & Needle stick \\
\hline $0(0.0 \%)$ & The \\
\hline $3(3.6 \%)$ & Other incident requiring supervisor assistance (describe): trouble locating point; trouble getting de qi; needed help keeping needle in skin with electrodes attached. \\
\hline
\end{tabular}

Table 3: Intern Reports of Errors and Events.

\begin{tabular}{|c|c|c|c|c|c|}
\hline \multicolumn{6}{|c|}{ Group Statistics } \\
\hline & & $\mathbf{N}$ & Mean & Std. Deviation & Std. Error Mean \\
\hline \multirow{2}{*}{ Gives you satisfactory treatment: } & No Electrical Stimulation & 51 & 4.76 & 0.737 & 0.103 \\
\hline & With Electrical Stimulation & 108 & 4.81 & 0.582 & 0.056 \\
\hline \multirow{2}{*}{ Feeling Relaxed } & No Electrical Stimulation & 52 & 0.75 & 0.437 & 0.061 \\
\hline & With Electrical Stimulation & 112 & 0.63 & 0.486 & 0.046 \\
\hline Feeling Energized & With Electrical Stimulation & 112 & 0.38 & 0.489 & 0.046 \\
\hline \multirow{2}{*}{ Feeling Drowsy } & No Electrical Stimulation & 52 & 0.12 & 0.323 & 0.045 \\
\hline & With Electrical Stimulation & 112 & 0.09 & 0.286 & 0.027 \\
\hline \multirow{2}{*}{ Feeling Pain } & No Electrical Stimulation & 52 & 0.13 & 0.345 & 0.048 \\
\hline & With Electrical Stimulation & 112 & 0.16 & 0.369 & 0.035 \\
\hline Cramping & No Electrical Stimulation & 52 & 0.04 & 0.194 & 0.027 \\
\hline \multirow{2}{*}{ Sweating } & No Electrical Stimulation & 52 & 0.02 & 0.139 & 0.019 \\
\hline & With Electrical Stimulation & 112 & 0.03 & 0.162 & 0.015 \\
\hline \multirow{2}{*}{ Feeling Faint } & No Electrical Stimulation & 52 & 0 & 0 & 0 \\
\hline & With Electrical Stimulation & 112 & 0.01 & 0.094 & 0.009 \\
\hline
\end{tabular}

Table 4: T-Test Analysis of Findings.

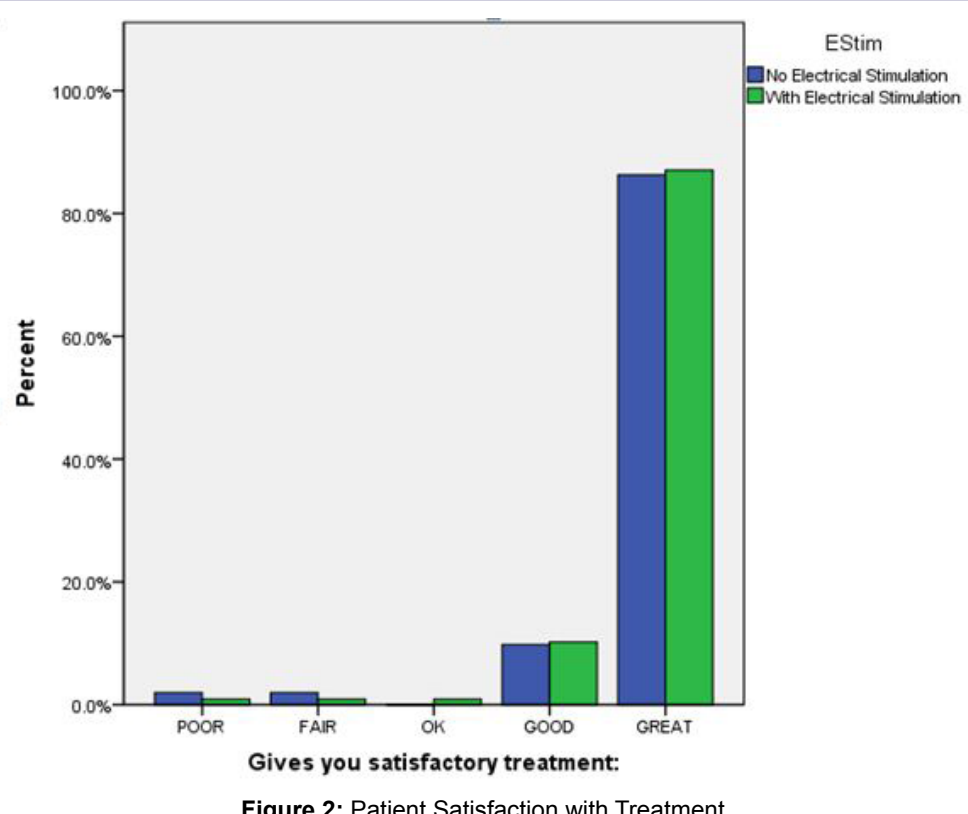

Figure 2: Patient Satisfaction with Treatment.

of being a teaching clinic or whether this is due to patient reticence about voicing concerns about treatment directly to those providing the treatment is unclear.
Comparison of the outcomes with other well-known studies of acupuncture safety (White 2002 and McPherson 2005) also demonstrated that there was more pain and cramping overall in the 
student clinical setting but otherwise the safety of electrical stimulation acupuncture compared well with standard acupuncture. Bleeding and bruising at the time of treatment were very similar (3.6-4.7\%). Patient satisfaction was high in all groups.

The total number of patients enrolled in this study was small, limiting the ability to show significant differences between the two groups. The small size also limited the likelihood of rare serious adverse events of acupuncture (local site infection, pneumothorax) from being seen in this population. However, due to similar outcomes with the McPherson \& White studies, there is a high likelihood that acupuncture with electrical stimulation compares favorably with needle only acupuncture in terms of safety and patient satisfaction.

\section{Conclusion}

Acupuncture with electrical stimulation compares favorably with needle only acupuncture in terms of both safety and patient satisfaction.

\section{References}

1. Ernst E, White AR (2001) Prospective studies of the safety of acupuncture: a systematic review. Am J Med 110: 481-485.

2. Lao L, Hamilton GR, Fu J, Berman BM (2003) Is acupuncture safe? A systematic review of case reports. AlternTher Health Med 9: 72-83.

3. White $A$ (2004) A cumulative review of the range and incidence of significant adverse events associated with acupuncture. Acupunct Med 22: 122-133.
4. Melchart D, Weidenhammer W, Streng A, Reitmayr S, Hoppe A, et al. (2004) Prospective investigation of adverse effects of acupuncture in 97,733 patients. Arch Intern Med 164: 104-105.

5. Park JE, Lee MS, Choi JY, Kim BY, Choi SM (2010) Adverse events Associated with Acupuncture: A Prospective Survey. J Altern Complement Med 16: $959-963$

6. Witt CM, Pach D, Brinkhaus B, Wruck K, Tag B, et al. (2009) Safety of acupuncture: results of a prospective observational study with 229,230 patients and introduction of a medical information and consent form. Forsch Komplementmed 16: 91-97.

7. Xu S, Wang L, Cooper E, Zhang M, Manheimer E, et al. (2013) Adverse Events of Acupuncture: A Systematic Review of Case Reports. Evid Based Complement Alternat Med 2013: 581203.

8. Xing M, Long AF (2006) A retrospective survey of patients at the University of Salford Acupuncture Clinic. Complement Ther Clin Pract 12: 64-71.

9. MacPherson $\mathrm{H}$, Thomas $\mathrm{K}$ (2005) Short term reactions to acupuncture--a cross-sectional survey of patient reports. Acupunct Med 23: 112-120.

10. White AR, Hayhoe S, Hart A, Ernst E (2001) Survey of Adverse events Following Acupuncture (SAFA): a prospective study of 32,000 consultations. Acupunct Med 19: 84-92.

11. Zhang J, Shang H, Gao X, Ernst E (2010) Acupuncture-related adverse events: a systematic review of the Chinese literature. Bull World Health Organ 88: 915-921. 